\title{
Joint Decoding and Estimation of Spatio-Temporally Correlated Binary Sources
}

\author{
Mohd Azri Mohd Izhar, Member, IEEE, Abdullah Jeza Aljohani, Member, IEEE, Soon Xin Ng, Senior \\ Member, IEEE, and Lajos Hanzo, Fellow, IEEE
}

\begin{abstract}
In the context of distributed joint source-channel coding, we conceive a joint decoding and estimation scheme for binary Markov sources exhibiting spatio-temporal correlation. The proposed scheme is designed based on the serial concatenation of a trellis coded modulation (TCM) scheme and a unityrate code. The symbol-based maximum a posteriori algorithm employed for TCM decoding is modified in order to exploit the source correlation. The estimation of both the spatial and temporal correlation parameters is performed jointly with the iterative decoding, hence allowing the estimated parameters to be updated after each iteration. Our simulation results reveal that when both the spatial and temporal correlation parameters are unknown, the proposed joint decoding and estimation scheme approaches the performance to the ideal system relying on perfectly known correlation parameters, therefore demonstrating the superiority of the proposed scheme.
\end{abstract}

Index Terms-Joint source-channel coding, distributed source coding, joint decoding and estimation.

\section{INTRODUCTION}

$\mathbf{U}$ NIFYING distributed source coding and joint sourcechannel coding, the proposed distributed joint sourcechannel coding (DJSCC) scheme offers an attractive solution for the transmission of correlated sources over noisy channels in practical finite-delay and finite-complexity systems [1]. The main goal of DJSCC is to minimize the energy required for reliable communications. In order to achieve a performance close to the Slepian-Wolf/Shannon limit, the related contributions to DJSCC have been mainly based on nearcapacity channel codes, such as turbo codes or turbo-like codes [1], [2], low-density parity-check (LDPC) codes [3], [4] and turbo trellis coded modulation (TTCM) [5]-[7]. In the abovementioned DJSCC contributions, the inter-source redundancy termed as spatial correlation is efficiently exploited by the channel decoder. However, the above contributions have assumed having independent and identically distributed (i.i.d) sources, which may not always be the case in real world scenarios, where the sources may exhibit temporal memory.

M. A. M. Izhar is with the Wireless Communication Center and UTM Razak School of Engineering and Advanced Technology, Universiti Teknologi Malaysia, Jalan Sultan Yahya Petra, Kuala Lumpur, 54100, Malaysia. E-mail: mohdazri.kl@utm.my.

A. J. Aljohani is with the Umm Alqura University, Mecca, Saudi Arabia. E-mail: ajra1c09@ecs.soton.ac.uk.

S. X. Ng and L. Hanzo are with the School of Electronics and Computer Science, University of Southampton, Southampton, SO17 1BJ, United Kingdom. Email: $\{$ sxn, lh $\} @$ ecs.soton.ac.uk.

This work was supported in part by the Malaysian Ministry of Higher Education, in part by the Universiti Teknologi Malaysia, in part by the European Research Council through the Advanced Fellow Grant, in part by the Royal Society's Wolfson Research Merit Award.
Hence, the authors of [8]-[10] exploited the spatio-temporal correlation of the sources and demonstrated that further performance improvements can be achieved by exploiting both the spatial and temporal correlations, as compared to spatial correlation only.

The accurate statistical knowledge of the correlation is important, since inaccurate statistical knowledge may potentially lead to catastrophic error propagation during the decoding process. In order to avoid this problem, it was assumed in [8][10] that the partial or full statistical knowledge of the spatiotemporal correlation is available at the receiver. However, this idealistic assumption can only be justified by relying on additional resources to forward the correlation coefficients to the receiver. Given that the source correlation tends to vary with time and space, the amount of additional resources needed may become significant. Online estimation of the correlation parameters is one of the promising solutions for practical DJSCC dispensing with the knowledge of correlation at the receiver. Estimation of the spatial-only correlation parameter has been addressed in [7], [8], [11] and high accuracy in estimating the spatial correlation was attained.

Against this background, we introduce a joint decoding and estimation scheme for the transmission of two spatiallycorrelated binary Markov sources exhibiting spatio-temporal correlation. We consider an asymmetric DSC scenario, where one of the two sources is transmitted over a noisy link and the other source is assumed to be perfectly available at the receiver as the side information to the first source. We employ a bandwidth-efficient DJSCC scheme constituted by a serialconcatenated trellis coded modulation (TCM) scheme [12] and a non-binary unity-rate code (URC) [13]. The serial concatenated TCM scheme and an URC can achieve better performance while having lower computational complexity than the TTCM-based DJSCC scheme [10]. The symbol-based maximum a posteriori (MAP) algorithm [14] is modified for TCM decoding in order to exploit the spatio-temporal source correlation, while performing iterative decoding. The estimation of the spatio-temporal correlation is also performed jointly with the iterative decoding. As a benefit, the spatiotemporal correlation parameters are updated at every decoding iteration. Hence, our novel contributions can be summarized as follows:

- We conceive a joint decoding and correlation estimation scheme for transmission of spatio-temporally correlated binary sources. In this contribution, the proposed scheme is based on an intrinsically amalgamated distributed joint source coding and URC-assisted TCM scheme. However, 
the proposed joint decoding and estimation scheme can also be generically applied to any channel code relying on MAP decoding.

- We propose a modified symbol-based MAP algorithm for TCM decoding to exploit the binary source correlation. We demonstrate the performance advantage of the joint trellis diagram design of the TCM scheme and the Markov process relying on the proposed MAP algorithm over the separate trellis design of the TCM scheme and the Markov process.

The remainder of this correspondence is organized as follows. The system model proposed for exploiting the spatiotemporal correlation using distributed joint source coding and URC-assisted TCM is described in Section II. The proposed joint decoding and estimation scheme is detailed in Section III. Our simulation results characterizing the scheme advocated are presented in Section IV, while our concluding remarks are provided in Section V.

\section{Distributed Joint Source Coding And URC-ASSISTED TCM FOR SPATIO-TEMPORALLY CORRElATED BINARY SOURCES}

A pair of binary source sequences having a length of $N_{\mathrm{b}}$ bits, namely $\mathbf{b}^{1}=\left\{b_{1}^{1}, b_{2}^{1}, \ldots, b_{N_{\mathrm{b}}}^{1}\right\}$ and $\mathbf{b}^{2}=\left\{b_{1}^{2}, b_{2}^{2}, \ldots, b_{N_{\mathrm{b}}}^{2}\right\}$ are considered, as illustrated in Fig. 1. The two sources are spatially correlated and the spatial correlation can be described at a time instant $t$ by the transition probabilities $a_{i, j}^{\mathrm{sp}}=P\left(b_{t}^{1}=\right.$ $\left.j \mid b_{t}^{2}=i\right)$ and $a_{i, j}^{\mathrm{sp} 2}=P\left(b_{t}^{2}=j \mid b_{t}^{1}=i\right)$ for the first source and second source, respectively where $i, j \in\{0,1\}$. The two sources are also temporally correlated, which is modeled by a first-order Markov process having transition probabilities of $a_{i, j}^{\mathrm{te}}=P\left(b_{t}^{1}=j \mid b_{t-1}^{1}=i\right)$ and $a_{i, j}^{\mathrm{te} 2}=P\left(b_{t}^{2}=j \mid b_{t-1}^{2}=i\right)$ for the first source and second source, respectively where $i, j \in$ $\{0,1\}$.

In our system model of Fig. 1, asymmetric DSC is assumed, where the first source is transmitted over a noisy link, while the second source is assumed to be readily available at the receiver as side information to the first source. The source bits $\mathbf{b}^{1}$ are encoded by a TCM encoder [12], $\mathrm{ENC}_{\mathrm{T}}$ to yield an $N_{\mathrm{s}}$-length symbol-sequence output of $\mathbf{v}=\left\{v_{1}, v_{2}, \ldots, v_{N_{\mathrm{s}}}\right\}$, which is then interleaved by a symbol interleaver $\Pi$ and encoded by a nonbinary URC encoder [13], $\mathrm{ENC}_{\mathrm{U}}$. The coded sequence $\mathbf{c}=$ $\left\{c_{1}, c_{2}, \ldots, c_{N_{\mathrm{s}}}\right\}$ is then modulated in order to form the signal $\mathbf{X}=\left\{x_{1}, x_{2}, \ldots, x_{N_{\mathrm{s}}}\right\}$ for transmission over an uncorrelated Rayleigh fading channel to yield the received sequence $\mathbf{y}=$ $\left\{y_{1}, y_{2}, \ldots, y_{N_{\mathrm{s}}}\right\}$ at the receiver. The received signal $y_{k}$ of the $k$-th symbol index of the sequence is given by

$$
y_{k}=h_{k} x_{k}+n_{k}
$$

where $h_{k}$ is a complex-valued channel coefficient and $n_{k}$ is a complex AWGN having a zero mean and a variance of $N_{0} / 2$ per dimension.

\section{A. Iterative decoding exploiting the spatio-temporal correla- tion}

At the receiver, iterative decoding is invoked for exchanging extrinsic information between the URC decoder $\left(\mathrm{DEC}_{\mathrm{U}}\right)$ and the TCM decoder $\left(\mathrm{DEC}_{\mathrm{T}}\right)$ for exploiting the spatio-temporal correlation and for jointly estimating the corresponding correlation parameters with the channel decoding, as shown in Fig. $1 . \mathrm{DEC}_{\mathrm{U}}$ receives the channel's symbol probability $Z(\mathbf{c})$ from the demapper as well as the a priori symbol probabilities $P^{\mathrm{a}}\left(\mathbf{v}^{\prime}\right)$ from $\mathrm{DEC}_{\mathrm{T}}$ and performs symbol-based MAP decoding to yield the extrinsic symbol probability outputs $P^{\mathrm{e}}\left(\mathbf{v}^{\prime}\right)$. This extrinsic information is then de-interleaved by $\Pi^{-1}$ to generate the a priori information $P^{\mathrm{a}}(\mathbf{v})$ for $\mathrm{DEC}_{\mathrm{T}}$. The side information gleaned from the second source, which is in the form of a priori probabilities $P^{\mathrm{SI}}\left(\mathbf{b}^{1}\right)$ is also fed to $\mathrm{DEC}_{\mathrm{T}}$, where $P^{\mathrm{SI}}\left(b_{t}^{1}\right)$ at a time instant $t$ aided by the function $f_{\mathrm{c}}$ is given by:

$$
P^{\mathrm{SI}}\left(b_{t}^{1}=j\right)=\sum_{i=0}^{1} \hat{a}_{i, j}^{\mathrm{sp}} \cdot P\left(b_{t}^{2}=i\right),
$$

where $\hat{a}_{i, j}^{\mathrm{sp}}$ represents the estimated transition probabilities characterizing the spatial correlation for $i, j \in\{0,1\}$. Based on both the a priori information, $P^{\mathrm{a}}(\mathbf{v})$ and $P^{\mathrm{a}}\left(\mathbf{b}^{1}\right)$ as well as on the estimated transition probabilities associated with the temporal correlation, $\hat{a}_{i, j}^{\mathrm{te}}$ for $i, j \in\{0,1\}$, the TCM decoder $\mathrm{DEC}_{\mathrm{T}}$ computes the a posteriori information $P^{\mathrm{o}}\left(\mathbf{b}^{1}\right)$, the joint a posteriori information $P^{\mathrm{o}}\left(b_{t-1}^{1}, b_{t}^{1}\right)$ for $t \in\left\{1,2, \ldots, N_{\mathrm{b}}\right\}$ and the extrinsic information $P^{\mathrm{e}}(\mathbf{v})$. The computation is performed using an appropriately modified version of the symbol-based MAP algorithm, as detailed in Section II-B, which takes into account both the side information $P^{a}\left(\mathbf{b}^{1}\right)$ and also the temporal memory inherent in the first source. The a posteriori information $P^{\mathrm{o}}\left(\mathbf{b}^{1}\right)$ is then used for computing the estimated spatial correlation parameter $\hat{a}_{i, j}^{\mathrm{sp}}$, meanwhile the a posteriori information $P^{\mathrm{o}}\left(\mathbf{b}^{1}\right)$ and the joint a posteriori information $P^{\mathrm{o}}\left(b_{t-1}^{1}, b_{t}^{1}\right)$ are used to compute the estimated temporal correlation parameter $\hat{a}_{i, j}^{\mathrm{te}}$, which will be further explained in Section-III. Since both the spatial and temporal correlations are unknown during the first iteration, we initialize $\hat{a}_{i, j}^{\mathrm{sp}}=\hat{a}_{i, j}^{\mathrm{te}}=0.5$ for $i, j \in\{0,1\}$. The extrinsic information $P^{\mathrm{e}}(\mathbf{v})$ output by $\mathrm{DEC}_{\mathrm{T}}$ is interleaved by $\Pi$ of Fig. 1 before being fed into the $\mathrm{DEC}_{\mathrm{U}}$ as the a priori information $P^{\mathrm{a}}\left(\mathbf{v}^{\prime}\right)$. The same process is repeated for a number of iterations and after the final iteration, a hard-decision is performed based on the a posteriori information $P^{\mathrm{o}}\left(\mathbf{b}^{1}\right)$ for obtaining the estimated bit-sequence $\hat{\mathbf{b}}^{1}$.

\section{B. Modification of the symbol-based MAP algorithm to exploit the binary source correlation}

The binary source correlation can be exploited by appropriately modifying the classic symbol-based MAP algorithm of [14] for TCM decoding. Explicitly, the modification of the symbol-based MAP algorithm required for TCM decoding can be derived by further developing the modified MAP algorithm of [15], [16] originally conceived for bit-based convolutional code MAP decoding. For an $M$-bit input symbol of TCM, the $N_{\mathrm{b}}$-bit length sequence of $\mathbf{b}^{1}$ can be represented as an $\left(N_{\mathrm{s}}=N_{\mathrm{b}} / M\right)$-symbol sequence $\mathbf{u}=\left\{u_{1}, u_{2}, \ldots, u_{k}, \ldots, u_{N_{\mathrm{s}}}\right\}$, where $u_{k}$ is a block of $M$ bits, i.e., we have $u_{k}=\left[u_{k, 1}, u_{k, 2}, \ldots, u_{k, l}, \ldots, u_{k, M}\right]$ and $u_{k, l}=$ $b_{M(k-1)+l}^{1}$. We have derived the a posteriori probabilities 


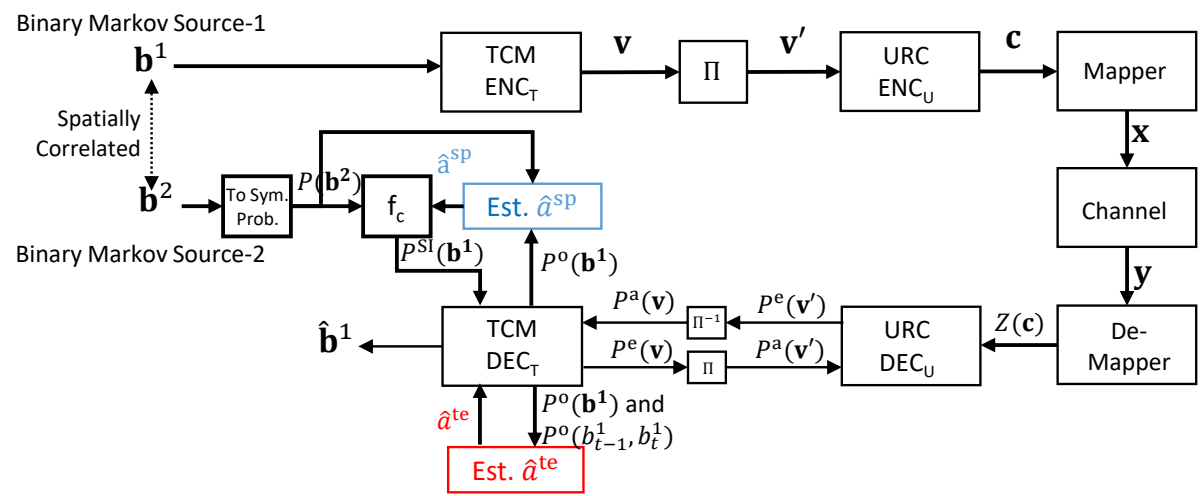

Fig. 1. Block diagram of a communication system with the proposed joint decoding and estimation scheme for spatio-temporally correlated binary sources.

associated with the input-symbol $u_{k}=e$, namely $P^{\mathrm{o}}\left(u_{k}=e\right)$ where $e \in\left\{0,1, \ldots, 2^{M}-1\right\}$ and that of the output-symbol $v_{k}=f$, namely $P^{\mathrm{o}}\left(v_{k}=f\right)$, where $f \in\left\{0,1, \ldots, 2^{M+1}-1\right\}$ taking into account the temporal bit-correlation as follows:

$$
\begin{aligned}
& P^{\mathrm{o}}\left(u_{k}=e\right)=P\left(u_{k}=e \mid \mathbf{y}\right) \\
&=\sum_{s^{\prime}=0}^{S-1} \sum_{i=0}^{1} \alpha_{k-1}\left(s^{\prime}, i\right) \cdot \beta_{k}\left[L_{s}\left(s^{\prime}, e\right), e_{M}\right] \\
& \cdot \gamma_{k}\left(s^{\prime}, i, e\right)
\end{aligned}
$$

and

$$
\begin{aligned}
P^{\mathrm{o}}\left(v_{k}=f\right) & =P\left(v_{k}=f \mid \mathbf{y}\right) \\
& =\sum_{e, s^{\prime} \in f} \sum_{i=0}^{1} \alpha_{k-1}\left(s^{\prime}, i\right) \cdot \beta_{k}\left[L_{s}\left(s^{\prime}, e\right), e_{M}\right] \\
\cdot \gamma_{k}\left(s^{\prime}, i, e\right) &
\end{aligned}
$$

where $i \in\{0,1\}$ and $e_{M} \in\{0,1\}$ are associated with the value of $u_{k-1, M}$ and $u_{k, M}$ respectively. Furthermore, $\alpha_{k}$ is the forward-recursive coefficient, $\beta_{k}$ is the backwardrecursive coefficient and $\gamma_{k}$ is the branch transition metric. The lookup table function $L_{s}\left(s^{\prime}, e\right)$ outputs the current trellis state $S_{k}=s$ from $S$ possible number of trellis states, as determined by the previous trellis state $S_{k-1}=s^{\prime}$ and by the input symbol $u_{k}=e$ The forward-recursive coefficient $\alpha_{k}$ and the backward-recursive coefficient $\beta_{k}$ are formulated as:

$$
\alpha_{t}\left(s, e_{M}\right)=\sum_{i=0}^{1} \alpha_{t-1}\left[L_{s^{\prime}}(s, e), i\right] \cdot \gamma_{t}\left[L_{s^{\prime}}(s, e), i, e_{M}\right]
$$

and

$$
\beta_{t-1}\left(s^{\prime}, i\right)=\sum_{i=0}^{1} \beta_{t}\left[L_{s}\left(s^{\prime}, e\right), e_{M}\right] \cdot \gamma_{t}\left(s^{\prime}, i, e_{M}\right),
$$

respectively, where $L_{s^{\prime}}(s, e)$ represents the lookup table outputting the previous trellis state $S_{k-1}=s^{\prime}$ determined by $S_{k}=s$ and $u_{k}=e$. The branch metric $\gamma_{k}$ is given by

$$
\begin{aligned}
\gamma_{k}\left(s^{\prime}, i, e\right)= & P^{\mathrm{a}}\left[v_{k}=L_{f}\left(s^{\prime}, e\right)\right] \cdot P^{\mathrm{SI}}\left(u_{k}=e\right) \\
& \cdot \hat{a}_{i, e_{1}}^{\mathrm{te}} \prod_{l=2}^{M} \hat{a}_{e_{l-1}, e_{l}}^{\mathrm{te}},
\end{aligned}
$$

where $e_{l}$ corresponds to the $l$-th bit value of $e, L_{f}\left(s^{\prime}, e\right)$ represents the lookup table outputting the coded symbol $v_{k}=f$ determined by the previous trellis state $S_{k-1}=s^{\prime}$ and by the input bit $u_{k}=e$, while $P^{\mathrm{SI}}\left(u_{k}\right)=\prod_{l=1}^{M} P^{\mathrm{SI}}\left(u_{k, l}\right)$ is the side information gleaned from the second source associated with the input symbol $u_{k}$ obtained from $P^{\mathrm{SI}}\left(\mathbf{b}^{1}\right)$. The marginal a posteriori probability $P^{\mathrm{o}}\left(b_{M(k-1)+l}^{1}\right)=P^{\mathrm{o}}\left(u_{k, l}\right)$ is then computed from $P^{\mathrm{o}}\left(u_{k}\right)$. The extrinsic probability $P^{\mathrm{e}}\left(v_{k}\right)$ can then be obtained by subtracting the a priori probability $P^{\mathrm{a}}\left(v_{k}\right)$ from the resultant a posteriori probability $P^{\mathrm{o}}\left(v_{k}\right)$.

\section{ESTIMATION OF THE SPATIO-TEMPORAL CORRELATION PARAMETERS}

In order to fully exploit the spatio-temporal correlation,it has to be accurately estimated. The transition probabilities associated with the spatial correlation, i.e. $a_{i, j}^{\mathrm{sp}}$ for $i, j \in\{0,1\}$ can be estimated by exploiting the a posteriori information $P^{\mathrm{o}}\left(\mathbf{b}^{1}\right)$ output by $\mathrm{DEC}_{\mathrm{T}}$ of Fig. 1 using the following formula [7]:

$$
\hat{a}_{i, j}^{\mathrm{sp}}=\frac{\sum_{t=1}^{N_{\mathrm{b}}} P^{\mathrm{o}}\left(b_{t}^{1}=j\right) \cdot P\left(b_{t}^{2}=i\right)}{\sum_{t=1}^{N_{\mathrm{b}}} P\left(b_{t}^{2}=i\right)} .
$$

Meanwhile, the estimation of the temporal correlation requires two sets of information, namely the a posteriori information $P^{\mathrm{o}}\left(\mathbf{b}^{1}\right)$ and the joint a posteriori information $P^{\mathrm{o}}\left(b_{t-1}^{1}, b_{t}^{1}\right)$. The joint a posteriori information $P^{\mathrm{o}}\left(b_{t-1}^{1}, b_{t}^{1}\right)$ can be obtained by first computing $P^{\mathrm{o}}\left(u_{k-1, M}, u_{k}\right)$ at a $k$-th symbol index from Eq. (3) as follows:

$$
\begin{aligned}
P^{\mathrm{o}}\left(u_{k-1, M}=i, u_{k}=e\right) & =\sum_{s^{\prime}=0}^{S-1} \alpha_{k-1}\left(s^{\prime}, i\right) \\
& \cdot \beta_{k}\left[L_{s}\left(s^{\prime}, e\right), e_{M}\right] \cdot \gamma_{k}\left(s^{\prime}, i, e\right) .
\end{aligned}
$$

Let us now represent $P^{\mathrm{o}}\left(u_{k-1, M}, u_{k}\right)$ as

$$
P^{\mathrm{o}}\left(b_{M(k-1)}^{1}, b_{M(k-1)+1}^{1}, b_{M(k-1)+2}^{1}, \ldots . ., b_{M(k-1)+M}^{1}\right) .
$$

The joint probability in Eq. (10) can then be marginalized into a set of joint probabilities of $P^{\mathrm{o}}\left(b_{M(k-1)}^{1}, b_{M(k-1)+1}^{1}\right), \quad P^{\mathrm{o}}\left(b_{M(k-1)+1}^{1}, b_{M(k-1)+2}^{1}\right)$, 
$\ldots \quad$ and $\quad P^{\mathrm{o}}\left(b_{M(k-1)+(M-1)}^{1}, b_{M(k-1)+M}^{1}\right)$. Finally, the transition probabilities corresponding to the temporal correlation can be estimated by:

$$
\hat{a}_{i, j}^{\mathrm{te}}=\frac{\sum_{t=2}^{N_{\mathrm{b}}} P^{\mathrm{o}}\left(b_{t-1}^{1}=i, b_{t}^{1}=j\right)}{\sum_{t=2}^{N_{\mathrm{b}}} P^{\mathrm{o}}\left(b_{t-1}^{1}=i\right)},
$$

for $i, j \in\{0,1\}$. The accuracy of the estimated transition probabilities is expected to be improved upon increasing the number of iterations and therefore, both $\hat{a}_{i, j}^{\mathrm{sp}}$ and $\hat{a}_{i, j}^{\mathrm{te}}$ for $i, j \in\{0,1\}$ are updated at every iteration.

\section{Simulation Results}

A series of computer simulations was carried out for evaluating the performance of the proposed joint decoding and estimation technique for sources exhibiting spatio-temporal correlation. The simulations were conducted using a frame length of $N_{\mathrm{b}}=20,000$ bits and considered symmetric Markov sources having the spatio-temporal correlation transition probabilities of:

$$
a_{i, j}^{\mathrm{sp}}= \begin{cases}p & \text { if } i=j, \\ 1-p & \text { if } i \neq j,\end{cases}
$$

and

$$
a_{i, j}^{\mathrm{te}}= \begin{cases}q & \text { if } i=j, \\ 1-q & \text { if } i \neq j,\end{cases}
$$

for $i, j \in\{0,1\}$ where $p$ and $q$ are the transition probabilities whose value is predetermined in order to generate sources exhibiting a specific spatial correlation and temporal correlation, respectively. We employed an 8-ary phase shift keying (PSK) 8 -state TCM scheme having a coding rate of $2 / 3$ and generator polynomial (in octal notation) of $\left[\begin{array}{lll}g_{r} & g_{1} & g_{2}\end{array}\right]_{8}=\left[\begin{array}{lll}13 & 2 & 4\end{array}\right]_{8}$, where $g_{r}$ is the feedback generator polynomial and $\left[\begin{array}{ll}g_{1} & g_{2}\end{array}\right]$ are the feed-forward generator polynomials. The number of decoding iterations was set to 20 and 5,000 frames were simulated at each signal-to-noise ratio (SNR) point.

We first evaluated the bit error ratio (BER) performance of the proposed MAP algorithm that is based on the combined trellis of the TCM scheme and of the Markov process to exploit the spatio-temporal correlation. The spatio-temporal correlation can also be exploited by considering a separate trellis design for TCM and Markov process, where the standard MAP algorithm can be employed for TCM decoding and iterative decoding is invoked for exchanging extrinsic information between three component decoders, namely $\mathrm{DEC}_{\mathrm{U}}, \mathrm{DEC}_{\mathrm{T}}$ and the Markov decoder. ${ }^{1}$ The decoding complexity is proportional to the product of the total number of trellis states (for all component decoders) and the number of iterations. In the proposed scheme, where the combined trellis of the TCM scheme and of the Markov process (joint TCM-Markov) is considered, the number of decoding iterations was set to 20 to result in a total complexity of $\propto\left(2^{2} \cdot 2+2^{3}\right) \times 20=320$ trellis states. For the sake of fair comparison in terms of complexity, 23 decoding iterations were invoked for the separate trellis

\footnotetext{
${ }^{1}$ The Markov decoder performs soft-bit source decoding of [17] to exploit the temporal correlation of a Markov process. The spatial correlation is also exploited by inputing the a priori information $P^{\mathrm{SI}}\left(\mathbf{b}^{1}\right)$ to the Markov decoder.
}

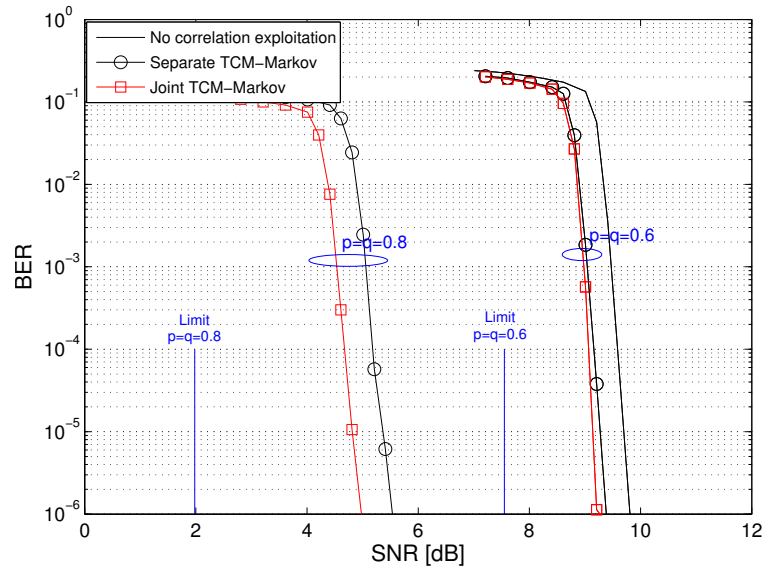

Fig. 2. Comparison in BER performance between the coding scheme based on the proposed joint TCM-Markov and the separate TCM-Markov designs for sources having $p=q=0.6$ and $p=q=0.8$, with both $p$ and $q$ are known at the receiver. Both of the schemes outperform the standard TCMURC scheme without exploiting any correlation. The vertical lines indicate the associated capacities [5], [10].

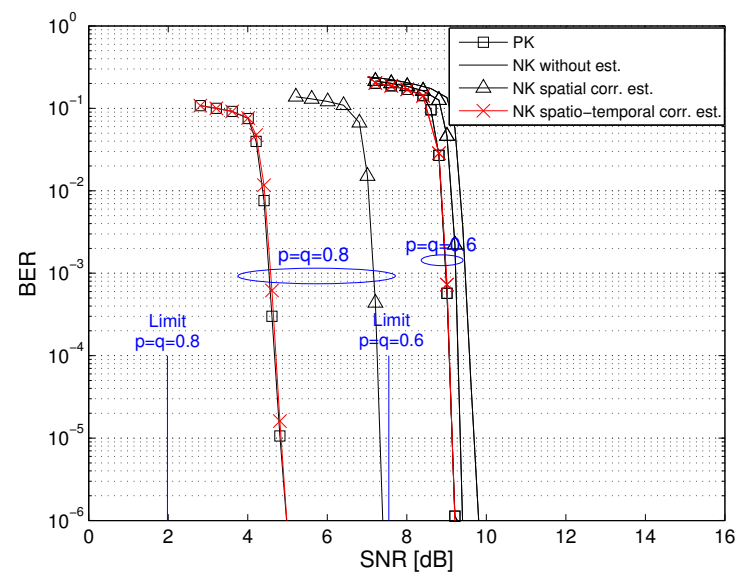

Fig. 3. BER performance of the proposed scheme estimating both the spatial and temporal correlation parameters, spatial correlation parameter only, and without estimating any correlation parameter when there is no knowledge (NK) of the correlation parameters at the receiver as compared to the ideal system with perfect knowledge (PK) of the correlation parameters at the receiver after 20 iterations for sources having $p=q=0.6$ and $p=q=0.8$. The vertical lines indicate the associated capacities [5], [10].

of TCM and Markov process (separate TCM-Markov), which yielded a complexity of $\propto\left(2+2^{2}+2^{3}\right) \times 23=322$ trellis states. It can be observed in Fig. 2 that the proposed scheme based on the joint TCM-Markov design outperforms the separate TCM-Markov for all the cases evaluated, i.e., for sources having $p=q=0.6$ and $p=q=0.8$ with $p$ and $q$ being assumed to be known at the receiver. The performance gap becomes more wide for sources having $p=q=0.8$ than for $p=q=0.6$, which suggests that the benefits of exploiting the spatio-temporal correlation using the proposed scheme based on joint TCM-Markov becomes more significant for stronger source correlation.

Based on the joint TCM-Markov design portrayed in Fig. 1, we then evaluated the performance of our proposed joint decoding and estimation scheme, when the correlation parameters are unknown at the receiver. Fig. 3 reveals that when the 


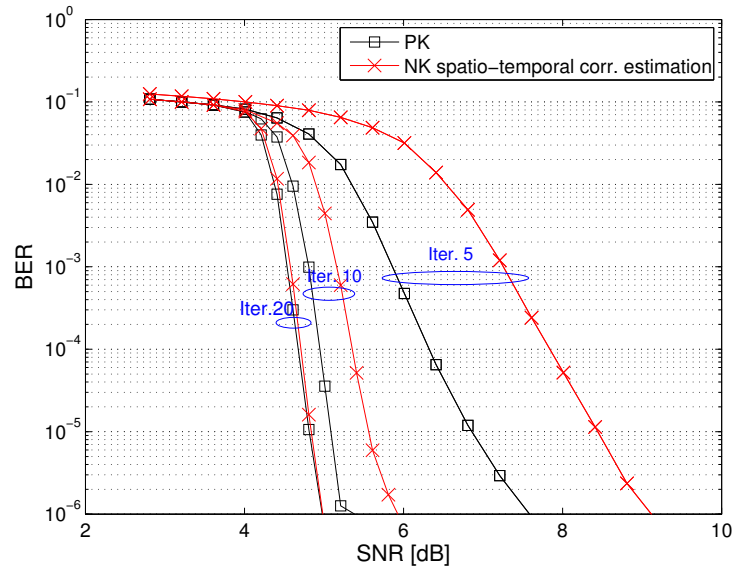

Fig. 4. BER performance of the proposed scheme when there is no knowledge (NK) of the correlation parameters at the receiver as compared to the ideal system with perfect knowledge (PK) of the correlation parameters at the receiver after 5,10 and 20 decoding iterations for sources having $p=q=0.8$.

correlation parameters are unknown at the receiver, the proposed joint decoding and estimation scheme outperforms both systems, namely the standard system operating without the estimation capability and the system, which can only estimate the spatial correlation for sources associated with $p=q=0.6$ and $p=q=0.8$. The performance gap was improved upon increasing the source correlation. Furthermore, we can observe that no performance loss is imposed on the proposed joint decoding and estimation scheme when the correlation parameters are unknown compared to the ideal system relying on the perfectly known correlation parameters. Therefore, a near-perfect estimation is demonstrated for the proposed scheme. However, when we invoked less decoding iterations, for example 5 iterations, the performance loss between the proposed joint decoding and estimation scheme having no knowledge of the correlation parameters and the ideal system having perfect knowledge becomes obvious, as depicted in Fig. 4. This is expected, since in the proposed scheme, we initially assume zero correlation regardless of the actual value of the correlation parameters during the first iteration. The accuracy of the estimated correlation parameters is gradually improved with the number of iterations. Thus, the proposed system is capable of achieving the same performance, as the ideal system at the cost of a higher number of decoding iterations.

\section{CONCLUSIONS}

In this correspondence, we have conceived a joint decoding and estimation scheme for binary sources exhibiting spatiotemporal correlation. The proposed scheme has been designed based on the serial concatenation of a TCM scheme and a symbol-based URC. A modified version of the symbolbased MAP algorithm was conceived for TCM decoding, which exploited the binary source correlation. Based on the proposed scheme, the estimation of both the spatial and temporal correlation parameters can be performed jointly with the iterative decoding and the estimated parameters can be updated after each iteration. Our simulation results have revealed that when the correlation parameters are unknown, our proposed joint decoding and estimation scheme achieves the same performance as the ideal system having perfect knowledge of the correlation parameters, demonstrating the accuracy of the proposed scheme.

\section{REFERENCES}

[1] J. Garcia-Frias and Y. Zhao, "Near-Shannon/Slepian-Wolf performance for unknown correlated sources over AWGN channels," IEEE Trans. Commun., vol. 53, no. 4, pp. 555-559, Apr. 2005.

[2] F. Daneshgaran, M. Laddomada, and M. Mondin, "Iterative joint channel decoding of correlated sources employing serially concatenated convolutional codes," IEEE Trans. Inform. Theory, vol. 51, no. 7, pp. 2721-2731, Jul. 2005.

[3] — "LDPC-based channel coding of correlated sources with iterative joint decoding," IEEE Trans. Commun., vol. 54, no. 4, pp. 577-582, Apr. 2006.

[4] I. Shahid and P. Yahampath, "Distributed joint source-channel coding using unequal error protection LDPC codes," IEEE Trans. Commun., vol. 61, no. 8, pp. 3472-3482, Aug. 2013.

[5] A. J. Aljohani, S. X. Ng, and L. Hanzo, "TTCM-aided rate-adaptive distributed source coding for Rayleigh fading channels," IEEE Trans. Veh. Technol., vol. 63, no. 3, pp. 1126-1134, Mar. 2014.

[6] _ - "Distributed source coding and its applications in relaying-based transmission," IEEE Access, vol. 4, pp. 1940-1970, 2016.

[7] A. Aljohani, Z. Babar, S. X. Ng, and L. Hanzo, "Distributed sourcechannel coding using reduced-complexity syndrome-based TTCM," IEEE Commun. Lett., vol. 20, no. 10, pp. 2095-2098, Oct. 2016.

[8] X. Zhou, M. Cheng, K. Anwar, and T. Matsumoto, "Distributed joint source-channel coding for relay systems exploiting sourcerelay correlation and source memory," EURASIP J. Wirel. Commun. Netw., vol. 2012, no. 1, pp. 1-13, 2012. [Online]. Available: http://dx.doi.org/10.1186/1687-1499-2012-260

[9] N. Sun, J. Wu, and G. Zhou, "Distributed joint source-channel code for spatial-temporally correlated Markov sources," in Communications (ICC), 2013 IEEE International Conference on, Jun. 2013, pp. 49054910.

[10] M. A. M. Izhar, A. J. Aljohani, S. X. Ng, and L. Hanzo, "Distributed joint source coding and trellis coded modulation for symbol-based Markov sources," submitted to IEEE TVT.

[11] Y. Zhao and J. Garcia-Frias, "Joint estimation and compression of correlated nonbinary sources using punctured turbo codes," IEEE Trans. Commun., vol. 53, no. 3, pp. 385-390, Mar. 2005.

[12] G. Ungerboeck, "Channel coding with multilevel/phase signals," IEEE Trans. Inform. Theory, vol. 28, no. 1, pp. 55-67, Jan. 1982.

[13] S. X. Ng, J. Wang, M. Tao, L. L. Yang, and L. Hanzo, "Iteratively decoded variable length space-time coded modulation: Code construction and convergence analysis," IEEE Trans. Wireless Commun., vol. 6, no. 5, pp. 1953-1963, May 2007.

[14] L. Hanzo, T. H. Liew, B. L. Yeap, R. Y. S. Tee, and S. X. Ng, Turbo Coding, Turbo Equalisation and Space-Time Coding: EXIT-Chart-Aided Near-Capacity Designs for Wireless Channels, 2nd Edition. New York, NY, USA: Wiley-IEEE Press, 2011.

[15] X. Zhou, K. Anwar, and T. Matsumoto, "Serially concatenated joint source-channel coding for binary Markov sources," in 6th Int. ICST Conf. Communications and Networking (CHINACOM), Harbin, China, 2011.

[16] M. A. Mohd Izhar, N. Fisal, X. Zhou, K. Anwar, and T. Matsumoto, "Exploitation of 2D binary source correlation using turbo block codes with fine-tuning," EURASIP J. Wirel. Commun. Netw., vol. 2013, no. 1, p. 89, Mar. 2013.

[17] Y. Huo, C. Zhu, and L. Hanzo, "Spatio-temporal iterative source-channel decoding aided video transmission," IEEE Trans. Veh. Technol., vol. 62, no. 4, pp. 1597-1609, May 2013. 